\title{
Title: A directed sampling approach for modelling the structural and electronic properties of amorphous alumina
}

Authors: A. F. Harper ${ }^{1}$, S. P. Emge' , P. C. M. M. Magusin² , C. P. Grey², Andrew J. Morris ${ }^{3, *}$

\section{Affiliations:}

${ }^{1}$ Theory of Condensed Matter, Cavendish Laboratory, University of Cambridge, J. J. Thomson Avenue, Cambridge CB3 0HE, U.K.

${ }^{2}$ Yusuf Hamied Department of Chemistry, University of Cambridge, Lensfield Road, Cambridge CB2 1EW, UK

${ }^{3}$ School of Metallurgy and Materials, University of Birmingham, Edgbaston, Birmingham B15 2TT, U.K

*a.j.morris.1@bham.ac.uk

\begin{abstract}
:
Advancing the next generation of materials for solid-state devices requires an understanding of their underlying electronic structure; yet the amorphous nature of many materials hinders progress in creating an atomic-level model. One such material is amorphous alumina, which is used to enhance performance in electronic devices from Li-ion batteries to perovskite solar cells. We develop a method which captures local properties of alumina through experimentally informed direct sampling of static configurations from ab initio molecular dynamics. This model is validated with solid-state nuclear magnetic resonance and X-ray absorption spectroscopy, calculated from the first principles level of theory. We finally construct an average electronic density of states for alumina from our model and observe two highly localized states at the conduction band edge.
\end{abstract}




\section{One Sentence Summary:}

Amorphous model construction by directed sampling, captures spectroscopic signatures of shortrange order and electronic properties.

\section{Main Text:}

Atomistic quantum mechanical modelling $(1,2)$ has played a critical role in driving many of the advances made involving crystalline materials over the last two decades - originally in terms of rationalizing materials properties, but now increasingly in predicting and optimizing both materials and devices. A significant challenge, however, lies in applying the same quantum mechanical methods to amorphous materials $(3,4)$, despite their increasing role in devices; these include dielectric layers in organic electronics, and as protective coatings across a range of materials (5-7). The challenge lies in capturing both the complexity of the local structure and the heterogeneity and structural variations that occur over a variety of length-scales. Amorphous structures lack the translational and point group symmetries present in crystalline materials, which enable calculations of increasingly large cells. It is also non-trivial to validate such a model against relevant experimental structural data, as symmetry allows structure solution from X-ray diffraction (XRD) experiments, while structure solution of amorphous materials relies on techniques such as Nuclear Magnetic Resonance (NMR) and X-ray Absorption Spectroscopy (XAS) to infer local structure. To understand and ultimately improve the electronic properties of an amorphous material, it is imperative to produce an accurate model of local atomic and electronic structure. 
Atomic layer deposited (ALD) alumina is one of the most widely used amorphous coating materials, as it is a model ALD system with well-understood surface chemistry $(8,9)$. ALD alumina was first employed as a high- $k$ dielectric and is present in applications across a range of electronic devices from solar cells to battery electrodes and field-effect transistors. Amorphous alumina $\left(\mathrm{a}-\mathrm{Al}_{2} \mathrm{O}_{3}\right)$ is a wide band-gap insulator which enables surface passivation, interface stability, and protects against degradation as a coating material $(5-7,10,11)$. These properties have increased the capacity retention of Li-ion battery electrodes (10-12), enhanced the lifetime of perovskite solar cells $(5,13)$, and improved the catalytic capabilities of metal-organic frameworks $(14,15)$. Given its clear widespread applications, an atomic level model of a- $\mathrm{Al}_{2} \mathrm{O}_{3}$ would be indispensable to the device physics community.

This work presents a method for modelling the local structure of an amorphous material at the quantum mechanical level of accuracy, which is then applied to $\mathrm{a}-\mathrm{Al}_{2} \mathrm{O}_{3}$. This method utilizes both first principles calculations and high-quality spectroscopy to confirm the detailed electronic structure of a- $\mathrm{Al}_{2} \mathrm{O}_{3}$. The novelty of our approach lies in the integration of locally sensitive experimental techniques with spectroscopy calculated from first principles We obtain NMR spectra at fields up to $1 \mathrm{GHz}$, which is the current state-of-the-art in solid state NMR and show that our DFT-based model contains the same structural features captured by these experimental measurements. The atomic level accuracy of this model enables us to identify two distinct fivefold coordination geometries present in $\mathrm{a}-\mathrm{Al}_{2} \mathrm{O}_{3}$, and the electronic states at the $\mathrm{Al}$ pre-edge of the absorption spectrum, which are unique to $\mathrm{a}-\mathrm{Al}_{2} \mathrm{O}_{3}$. We calculate an average electronic density of states on our model, in a computationally efficient and accurate manner, thereby opening the door for further investigation into amorphous electronic structure. 


\section{Amorphous model generation}

Building an amorphous model typically involves a tradeoff between large supercells and low interatomic accuracy, or small cell sizes and a limited description of the structure. Intuitively, a highaccuracy large cell size model is the goal, however first principles quantum mechanical calculations using density-functional theory (DFT) scale as $O\left(N^{3}\right)$ making these large cell size accurate calculations unfeasible. Our method, outlined in Fig. 1, uses experimentally driven directed sampling across a set of high-quality small cell size ab initio molecular dynamics (AIMD) calculations in order to construct a model which reproduces experimentally known spectroscopic signatures. The resultant model is a set of static configurations from AIMD which contains local orderings that are representative of the amorphous solid.

To build this model, we first generate a large set of AIMD simulations using a melt-quench technique which span a range of initial conditions (densities, equilibration temperatures, and rate of equilibration) as outlined in Fig. 1 Structure Generation. For each set of initial conditions, the AIMD simulations are repeated from different starting structures, thereby exploring additional local structural orderings. From these simulations, the static configurations from the final equilibrated 1000 timesteps of each simulation are considered. For the $\mathrm{a}-\mathrm{Al}_{2} \mathrm{O}_{3}$ model, we generated 18 initial conditions, with 3 starting structures each to generate a total of 54,000 static configurations. In this Structure Generation we have thus captured both the ergodicity across the range of timesteps, and the randomness across the range of AIMD simulations for $\mathrm{a}-\mathrm{Al}_{2} \mathrm{O}_{3}$. 
We further curate this data using experimental information in a process known as Fingerprinting (Fig. 1). Each set of data at a given initial condition is averaged across the 3 AIMD simulations for that set of initial conditions (a total of 3000 static configurations for each set of initial conditions). From this data, the concentrations of Al coordination environments, four- fiveand six-fold $\mathrm{Al}$, are extracted and compared to experimental values of $49 \%$ to $38 \%$ to $11 \%$ concentration, respectively (Fig. S1, Fig. S2). All sets of initial conditions that do not meet the tolerances of the experimental concentrations are then excluded, in order to determine which set of initial conditions will capture the bulk properties of a- $\mathrm{Al}_{2} \mathrm{O}_{3}$; this is our way of using directed sampling the configuration space for the static configurations that are most likely to capture experimental properties. Fingerprinting identifies two sets of initial conditions for a- $\mathrm{Al}_{2} \mathrm{O}_{3}$ that produce models with ratios of four-, five-, and six-fold Al coordination environments within error of the experimental data.

We now construct two amorphous models, one from each set of initial conditions which met the Fingerprinting criteria, as a set of randomly selected static configurations. For each initial condition, a set of 15 configurations across the final 1000 timesteps are randomly chosen from each of the 3 AIMD simulations, to create a total of 45 static configurations which make up the amorphous model. For some desired property, $X$, such as electronic density of states, we can calculate the average value of this property across these 45 static configurations $\left(N_{c}\right)$ as,

$$
X(N)=\sum_{c=1}^{45} X\left(N_{c}\right)
$$

This is analogous to an ensemble average of the property in which the probability of each configuration is equally likely, as we are in an amorphous solid with no symmetry equivalence. 
Herein we show that this amorphous model not only captures global properties such as coordination number, but also the specific local electronic properties predicted by experimental spectroscopy.

\section{Experimental Validation using First Principles Spectroscopy}

A set of 45 configurations is a tractable number of first principles spectroscopic calculations to carry out for the amorphous model. By using the method outlined in Eq. 1, the average NMR and XAS spectra were calculated, and compared to experimental spectroscopy on a 1000 layer sample of ALD deposited a- $\mathrm{Al}_{2} \mathrm{O}_{3} .{ }^{1}$ Reproducing these experimental signatures indicates that this model captures the local order, and electronic properties of the experimental amorphous phase.

The experimental ${ }^{27} \mathrm{Al}$ 1D NMR spectrum shown in Fig. 2A shows 3 distinct peaks corresponding to disordered four-, five-, and six-fold coordinated (Al(IV), Al(V) and Al(VI)) Al environments with a ratio of $49 \%$ to $38 \%$ to $11 \% \pm 2 \%$ respectively. The presence of the $\mathrm{Al}(\mathrm{V})$ signal at $40 \mathrm{ppm}$ and the low intensity of the $\mathrm{Al}(\mathrm{VI})$ signal at $11 \mathrm{ppm}$ are strong indicators of the amorphous nature (16). It is expected that a distribution of asymmetric Al environments, combined with the quadrupolar nature of the ${ }^{27} \mathrm{Al}$ nucleus $(I=5 / 2)$, would lead to large quadrupolar coupling constants $\left(C_{Q}\right)$ and a wide range of isotropic chemical shifts, as evidenced by the broad peaks shown in the 1D NMR spectrum in Fig. 2A. The 2D MQMAS NMR shows three signals spreading along the isotropic diagonal line and horizontally along the MAS dimension,

\footnotetext{
${ }^{1}$ In the main text, we show results for one amorphous model $\left(300 \mathrm{~K}, 3.18 \mathrm{~g} / \mathrm{cm}^{3}\right.$ initial conditions), with an equivalent set of results for the additional amorphous model $\left(600 \mathrm{~K}, 3.18 \mathrm{~g} / \mathrm{cm}^{3}\right.$ initial conditions) shown in the Supplementary Information.
} 
highlighting the spread of quadrupolar coupling constants and a chemical shift distribution (Fig. 2B).

The NMR isotropic shieldings for all 2160 atoms in the amorphous model are calculated using DFT GIPAW NMR and reproduce the total isotropic range and location of shifts in the experimental 1D spectrum (Fig. 2C). A wide distribution of $\mathrm{C}_{\mathrm{Q}}$ values and calculated quadrupolar induced shifts $\left(\delta_{C_{Q}}\right)$ across $\mathrm{Al}$ sites which ranges from 10 to $20 \mathrm{MHz}$ (Fig. 2D), with a wider range for both $\mathrm{Al}(\mathrm{IV})$ and $\mathrm{Al}(\mathrm{V})$ environments. The calculated isotropic shieldings and corresponding quadrupolar shifts show similar trends to experiment, indicating that the model captures these features from NMR.

The spin-simulation tool SIMPSON (17) was used to incorporate quadrupolar interactions at three fields $(11.75 \mathrm{~T}, 16.44 \mathrm{~T}, 23.49 \mathrm{~T})$ corresponding to the three fields at which the experimental NMR spectra (Fig. 3A) were obtained. The resulting spectra are shown in Fig. 3B and the quadrupolar nature of the $\mathrm{Al}(\mathrm{IV})$ and $\mathrm{Al}(\mathrm{V})$ environments is well described as evidenced by the sloping tails towards lower $\delta_{i s o}$ values, which are indicators of quadrupolar environments. Furthermore, trends at increasing field strengths are captured by both theory and experiment, with higher fields showing sharper peaks of the $\mathrm{Al}(\mathrm{IV}, \mathrm{V}, \mathrm{VI})$ environments, and a narrower overall spectral width.

One advantage of calculating the NMR spectrum from first principles, is that the calculations retain an individual spectrum for each atom in the model. In this way, we are able to construct spectra based on atom-specific coordination environments. Using a crystal-symmetry 
metric (CSM) (18), a measure of the relative distortion from perfect symmetry was extracted for each site in the model. A comparison of the CSM to isotropic chemical shift (Fig. 4A), shows that the majority of tetrahedral sites are distorted $(\mathrm{CSM}>4)(18)$, which agrees with the wide quadrupolar distribution of Al(IV) shifts in experimental MQMAS. Separating the spectra into the closest geometric environment, as shown in Fig. 3, determines that within the a- $\mathrm{Al}_{2} \mathrm{O}_{3}$, there exist two types of $\mathrm{Al}(\mathrm{V})$ environments, square pyramidal and trigonal bipyramidal; these sites combine to make the $\mathrm{Al}(\mathrm{V})$ peak in the $1 \mathrm{D} \mathrm{NMR}$ at $40 \mathrm{ppm}$, with a range of site-specific shifts from 20 to $60 \mathrm{ppm}$. Given $\mathrm{Al}(\mathrm{V}) \mathrm{s}$ unique appearance in amorphous $\mathrm{Al}_{2} \mathrm{O}_{3}$ phases, this identification of two geometries of $\mathrm{Al}(\mathrm{V})$ sites suggests that further work should involve identifying the role of each geometry in electronic devices. The first principles model of a- $\mathrm{Al}_{2} \mathrm{O}_{3}$ enables the construction of these two sites' spectra, where experimental NMR shows them as overlapping.

NMR is a method available for validating the local atomic structure while XAS is a measurement for probing the local electronic structure of $\mathrm{a}-\mathrm{Al}_{2} \mathrm{O}_{3}$. The $\mathrm{Al}$ K-edge XAS spectrum shown in Fig. 5, exhibits three main features; a pre-edge feature (a) and two dominant broad peaks at $1565 \mathrm{eV}$ (b) and $1570 \mathrm{eV}$ (c) which are similar to those in Al-rich glasses (19) and attributed to transitions in $\mathrm{Al}(\mathrm{IV})$ and $\mathrm{Al}(\mathrm{VI})$ respectively. The absorption edge for $\mathrm{Al}(\mathrm{V})$ lies between $\mathrm{Al}(\mathrm{IV})$ and $\mathrm{Al}(\mathrm{VI})$, and has no experimental reference. Calculating core-hole spectra for all $\mathrm{Al}$ sites in the amorphous model, determines the location of this $\mathrm{Al}(\mathrm{V})$ absorption edge between 1565 and 1570 $\mathrm{eV}$ (Fig. 5), and confirms the absorption energy of the $\mathrm{Al}(\mathrm{IV})$ and $\mathrm{Al}(\mathrm{VI})$ peaks, implying that the model's electronic structure is consistent with the experimental a- $\mathrm{Al}_{2} \mathrm{O}_{3}$. 
In addition to identifying the two main XAS peaks, the pre-peak at $1563 \mathrm{eV}$ was also reproduced. A pre-edge is documented for $\alpha-\mathrm{Al}_{2} \mathrm{O}_{3}$, at $1565 \mathrm{eV}$, which occurs as a result of transitions from the $1 s$ to $3 s$ states in $\mathrm{Al}(20)$. While this transition is normally dipole forbidden, the presence of atomic vibrations results in distorted octahedral environments and bond lengthening which causes $p$-s mixing to occur and allow this transition. The pre-edge in a- $\mathrm{Al}_{2} \mathrm{O}_{3}$ is at 1562 to $1563 \mathrm{eV}$, both in experiment and in the calculated spectra, as shown in Fig. 5, and occurs in either tetrahedral $\mathrm{Al}(\mathrm{IV})$ sites or square pyramidal $\mathrm{Al}(\mathrm{V})$ sites. Similarly to the $\alpha-\mathrm{Al}_{2} \mathrm{O}_{3}$ case, this pre edge is a result of site distortions at the Al(IV) site (Fig. S10) (20). The calculated XAS spectra enabled the discovery of the origins of this pre-edge feature as well as the absorption energy of the $\mathrm{Al}(\mathrm{V})$ peaks, which are masked in experiment.

\section{Implications of the model}

Previous work $(21,22)$ implies that amorphous models require thousands of atoms in the unit cell or semi-infinite simulation times to fully capture local properties. We show that by averaging across AIMD simulations from directed sampling, we produce a model of a- $\mathrm{Al}_{2} \mathrm{O}_{3}$ which exhibits the same local structural properties as captured by experimental NMR and the same electronic properties as captured by the XAS. We now extend this method to a first principles specific technique, the electronic density of states.

The electronic density of states (eDOS) for a- $\mathrm{Al}_{2} \mathrm{O}_{3}$ are of particular interest, as this material is a coating layer in electronic devices such as Li-ion batteries, semiconductors, and fieldeffect transistors. The eDOS, calculated as in Eq. 1, as an average across the 45 configurations in 
the amorphous model (Fig. 6), confirms the experimentally predicted wide band gap insulating nature of this material, with a calculated band gap of $2.6 \mathrm{eV}$ using the PBE functional. States at the valence band maximum are $\mathrm{O} p$ type character, and states at the bottom of the conduction band are $\mathrm{Al} s$ character. Interestingly, we identify two small peaks at the bottom of the conduction band at 3.2 and $4.2 \mathrm{eV}$ which are low density, but highly localized states in this material. These shallow states are unique features of the amorphous phase of $\mathrm{Al}_{2} \mathrm{O}_{3}$ which lead to the sharp pre-edge features in the XAS (Fig. 5).

Given the moderate system size, and open-source availability of the computing methods used herein, producing amorphous eDOS, and in turn NMR and XAS spectra is certainly achievable for other amorphous systems. The method of averaging across static configurations from experimentally directed sampling, with a moderate cell size AIMD simulation can be applied to other amorphous systems and used to calculate average properties of an amorphous material that are otherwise unattainable using large system sizes. We expect that given the transferability of this method, calculating these spectral properties, and potentially others will become the norm for simulations on amorphous solids, and especially in the field of device physics, encourage progress in fine tuning the electronic properties of these materials.

\section{References and Notes:}

1. J. Hoja, H.-Y. Ko, M. A. Neumann, R. Car, R. A. DiStasio Jr., A. Tkatchenko, Reliable and practical computational description of molecular crystal polymorphs. Science Advances. 45 (2019), doi:10.1126/SCIADV.AAU3338. 
2. W. SM, C. R, Crystal structure prediction from first principles. Nature Materials. 7, 937946 (2008).

3. M. Aykol, S. S. Dwaraknath, W. Sun, K. A. Persson, Thermodynamic limit for synthesis of metastable inorganic materials. Science Advances. 4 (2018), doi:10.1126/SCIADV.AAQ0148.

4. V. L. Deringer, N. Bernstein, G. Csányi, C. ben Mahmoud, M. Ceriotti, M. Wilson, D. A. Drabold, S. R. Elliott, Origins of structural and electronic transitions in disordered silicon. Nature 2020 589:7840. 589, 59-64 (2021).

5. C. Das, M. Kot, T. Hellmann, C. Wittich, E. Mankel, I. Zimmermann, D. Schmeisser, M. Khaja Nazeeruddin, W. Jaegermann, Atomic Layer-Deposited Aluminum Oxide Hinders Iodide Migration and Stabilizes Perovskite Solar Cells. Cell Reports Physical Science. 1, $100112(2020)$.

6. Z. Yang, T. Albrow-Owen, H. Cui, J. Alexander-Webber, F. Gu, X. Wang, T. C. Wu, M. Zhuge, C. Williams, P. Wang, A. v. Zayats, W. Cai, L. Dai, S. Hofmann, M. Overend, L. Tong, Q. Yang, Z. Sun, T. Hasan, Single-nanowire spectrometers. Science. 365, 10171020 (2019).

7. J. Lu, B. Fu, M. C. Kung, G. Xiao, J. W. Elam, H. H. Kung, P. C. Stair, Coking- and sintering-resistant palladium catalysts achieved through atomic layer deposition. Science. 335, 1205-1208 (2012).

8. R. L. Puurunen, Surface chemistry of atomic layer deposition: A case study for the trimethylaluminum/water process. Journal of Applied Physics. 97, 121301 (2005). 
9. S. M. George, Atomic Layer Deposition: An Overview. Chemical Reviews. 110, 111-131 (2009).

10. Y. Zhao, M. Amirmaleki, Q. Sun, C. Zhao, A. Codirenzi, L. v. Goncharova, C. Wang, K. Adair, X. Li, X. Yang, F. Zhao, R. Li, T. Filleter, M. Cai, X. Sun, Natural SEI-Inspired Dual-Protective Layers via Atomic/Molecular Layer Deposition for Long-Life Metallic Lithium Anode. Matter. 1, 1215-1231 (2019).

11. E. M. Lotfabad, P. Kalisvaart, K. Cui, A. Kohandehghan, M. Kupsta, B. Olsen, D. Mitlin, ALD $\mathrm{TiO}_{2}$ coated silicon nanowires for lithium ion battery anodes with enhanced cycling stability and coulombic efficiency. Physical Chemistry Chemical Physics. 15, 1364613657 (2013).

12. Y. Zhao, L. v. Goncharova, A. Lushington, Q. Sun, H. Yadegari, B. Wang, W. Xiao, R. Li, X. Sun, Superior Stable and Long Life Sodium Metal Anodes Achieved by Atomic Layer Deposition. Advanced Materials. 29, 1606663 (2017).

13. C.-Y. Chang, K.-T. Lee, W.-K. Huang, H.-Y. Siao, Y.-C. Chang, High-Performance, AirStable, Low-Temperature Processed Semitransparent Perovskite Solar Cells Enabled by Atomic Layer Deposition. Chemistry of Materials. 27, 5122-5130 (2015).

14. Z. Gao, Y. Qin, Design and Properties of Confined Nanocatalysts by Atomic Layer Deposition. Accounts of Chemical Research. 50, 2309-2316 (2017).

15. B. J. O’Neill, D. H. K. Jackson, J. Lee, C. Canlas, P. C. Stair, C. L. Marshall, J. W. Elam, T. F. Kuech, J. A. Dumesic, G. W. Huber, Catalyst Design with Atomic Layer Deposition. ACS Catalysis. 5, 1804-1825 (2015). 
16. S. K. Lee, S. Y. Park, Y. S. Yi, J. Moon, Structure and Disorder in Amorphous Alumina Thin Films: Insights from High-Resolution Solid-State NMR. Journal of Physical Chemistry C. 114, 13890-13894 (2010).

17. M. Bak, J. T. Rasmussen, N. C. Nielsen, SIMPSON: A general simulation program for solid-state NMR spectroscopy. J. Magn. Reson. 213, 366-400 (2011).

18. D. Waroquiers, X. Gonze, G.-M. Rignanese, C. Welker-Nieuwoudt, F. Rosowski, M. Göbel, S. Schenk, P. Degelmann, R. André, R. Glaum, G. Hautier, Statistical Analysis of Coordination Environments in Oxides. Chemistry of Materials. 29, 8346-8360 (2017).

19. D. R. Neuville, L. Cormier, D. Massiot, Al environment in tectosilicate and peraluminous glasses: A 27Al MQ-MAS NMR, Raman, and XANES investigation. Geochimica et Cosmochimica Acta. 68, 5071-5079 (2004).

20. D. Cabaret, C. Brouder, Origin of the pre-edge structure at the Al K-edge: The role of atomic vibrations. Journal of Physics: Conference Series. 190, 012003 (2009).

21. D. VL, B. N, B. AP, C. MJ, K. RN, M. LE, G. CP, E. SR, C. G, Realistic Atomistic Structure of Amorphous Silicon from Machine-Learning-Driven Molecular Dynamics. The journal of physical chemistry letters. 9, 2879-2885 (2018).

22. G. Sivaraman, A. N. Krishnamoorthy, M. Baur, C. Holm, M. Stan, G. Csányi, C. Benmore, Á. Vázquez-Mayagoitia, Machine-learned interatomic potentials by active learning: amorphous and liquid hafnium dioxide. npj Computational Materials 2020 6:1. 6, 1-8 (2020).

23. G. Engelhardt, H. Koller, A simple procedure for the determination of the quadrupole interaction parameters and isotropic chemical shifts from magic angle spinning NMR 
spectra of half-integer spin nuclei in solids. Magnetic Resonance in Chemistry. 29, 941945 (1991).

24. M. Mais, S. Paul, N. S. Barrow, J. J. Titman, Dynamic Nuclear Polarisation Enhanced Solid-State Nuclear Magnetic Resonance Studies of Surface Modification of $\gamma$-Alumina. Johnson Matthey Technology Review. 62, 271-278 (2018).

25. R. J. Nicholls, A. J. Morris, C. J. Pickard, J. R. Yates, OptaDOS - a new tool for EELS calculations. Journal of Physics: Conference Series. 371, 012062 (2012).

26. A. J. Morris, R. J. Nicholls, C. J. Pickard, J. R. Yates, OptaDOS: A tool for obtaining density of states, core-level and optical spectra from electronic structure codes. Computer Physics Communications. 185, 1477-1485 (2014).

27. J. C. C. Chan, H. Eckert, High-resolution 27Al-19F solid-state double resonance NMR studies of AlF3-BaF2-CaF2 glasses. Journal of Non-Crystalline Solids. 284, 16-21 (2001).

28. G. Kresse, J. Furthmüller, Efficient iterative schemes for ab initio total-energy calculations using a plane-wave basis set. Physical Review B. 54, 11169 (1996).

29. D. J. Evans, B. L. Holian, The Nose-Hoover thermostat. The Journal of Chemical Physics. 83, 4069 (1998).

30. P. E. Blöchl, Projector augmented-wave method. Physical Review B. 50, 17953 (1994).

31. L. Martínez, R. Andrade, E. G. Birgin, J. M. Martínez, PACKMOL: A package for building initial configurations for molecular dynamics simulations. Journal of Computational Chemistry. 30, 2157-2164 (2009). 
32. S. J. Clark, M. D. Segall, C. J. Pickard, P. J. Hasnip, M. I. J. Probert, K. Refson, M. C. Payne, First principles methods using CASTEP. Zeitschrift für Kristallographie Crystalline Materials. 220, 567-570 (2005).

33. C. J. Pickard, F. Mauri, All-electron magnetic response with pseudopotentials: NMR chemical shifts. Physical Review B. 63, 245101 (2001).

34. S.-P. Gao, C. J. Pickard, A. Perlov, V. Milman, Core-level spectroscopy calculation and the plane wave pseudopotential method. Journal of Physics: Condensed Matter. 21, 104203 (2009).

35. J. R. Yates, X. Wang, D. Vanderbilt, I. Souza, Spectral and Fermi surface properties from Wannier interpolation. Physical Review B. 75, 195121 (2007).

36. T. Mizoguchi, I. Tanaka, S.-P. Gao, C. J. Pickard, First-principles calculation of spectral features, chemical shift and absolute threshold of ELNES and XANES using a plane wave pseudopotential method. Journal of Physics: Condensed Matter. 21, 104204 (2009).

37. B. Karasulu, S. P. Emge, M. F. Groh, C. P. Grey, A. J. Morris, Al/Ga-doped $\mathrm{Li}_{7} \mathrm{La}_{3} \mathrm{Zr}_{2} \mathrm{O}_{12}$ Garnets as Li-ion solid-state battery electrolytes: atomistic insights into local coordination environments and their influence on 17O, 27Al, and 71Ga NMR spectra. Journal of the American Chemical Society. 142, 3132-3148 (2020).

38. T. Bräuniger, C. V. Chandran, U. Wedig, M. Jansen, NMR chemical shift and quadrupolar interaction parameters of carbon-coordinated $27 \mathrm{Al}$ in aluminium carbide, $\mathrm{Al}_{4} \mathrm{C}_{3}$. Zeitschrift fur Anorganische und Allgemeine Chemie. 637, 530-535 (2011).

39. D. Müller, W. Gessner, G. Scheler, Chemical shift and quadrupole coupling of the ${ }^{27} \mathrm{Al}$ NMR spectra of $\mathrm{LiAIO}_{2}$ polymorphs. Polyhedron. 2, 1195-1198 (1983). 
Acknowledgements: The authors thank Steve Haws (Henry Royce Institute, Cambridge) for assistance with the ALD and Richard Chen (University of Cambridge, Chemistry) for NMR sample preparation. The authors also thank Dr Trent Franks and the UK High-Field Solid-State NMR Facility Warwick for measurements on the $1 \mathrm{GHz}$ magnet. Funding: AFH acknowledges the financial support of the Gates Cambridge Trust and the Winton Programme for the Physics of Sustainability, University of Cambridge, UK. AJM acknowledges funding from EPSRC (EP/P003532/1). The authors acknowledge networking support via the EPSRC Collaborative Computational Projects, CCP9 (EP/M022595/1) and CCP-NC (EP/T026642/1). This work was performed using resources provided by the Cambridge Service for Data Driven Discovery (CSD3) operated by the University of Cambridge Research Computing Service (www.csd3.cam.ac.uk), provided by Dell EMC and Intel using Tier-2 funding from the EPSRC (capital grant EP/P020259/1), and DiRAC funding from the Science and Technology Facilities Council (www.dirac.ac.uk). SPE acknowledges funding via an EPSRC iCASE (Award 1834544) and via the Royal Society (RP $\backslash R 1 \backslash 180147)$. For ALD sample preparation, use of the Ambient Processing Cluster Tool, part of Sir Henry Royce Institute - Cambridge Equipment, EPSRC grant EP/P024947/1 is gratefully acknowledged. Author contributions: Angela F. Harper: Formal analysis, Writing - Original draft preparation, Data curation, Conceptualization. Steffen E. Emge: Investigation, Writing - review and editing. Pieter C. M. M. Magusin: Support with MQMAS setup and analysis, organization of $1 \mathrm{GHz}$ measurements. Clare P. Grey: Supervision, Resources, Writing - review and editing. Andrew J. Morris: Supervision, Resources, Writingreview and editing. Competing interests: The authors declare no competing interests. Data and Materials availability: All data and materials are available on request.

\section{Supplementary Materials:}

Materials and Methods

Supplementary Text

Figures S1-S12

Tables S1-S4

References (24-39) 


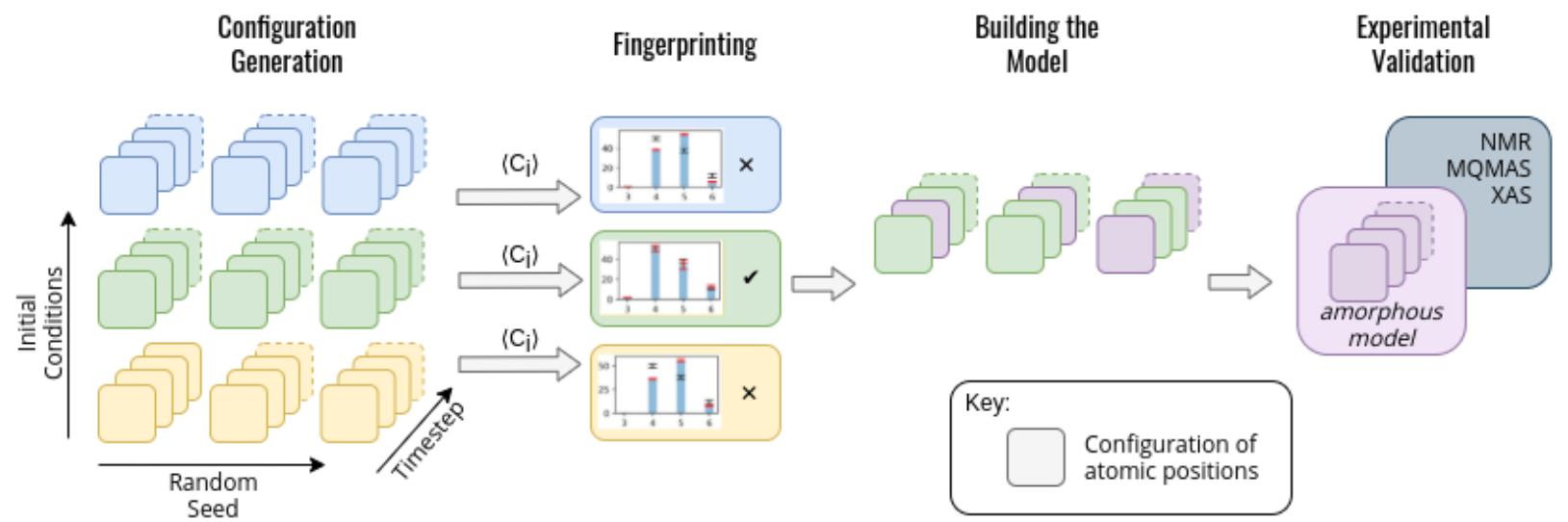

Fig. 1. Method for generating an amorphous model from a series of static configurations from AIMD. Structure Generation: Each colored box represents a single static configuration from an AIMD simulation, and their depth into the page shows the number of configurations over time (timestep arrow). These configurations are colored by their initial conditions (temperature, density, and rate of equilibration) such that blue, green, and yellow are three different sets of initial conditions. The three repeated sets of configurations represent repeated AIMD simulations with different randomly seeded starting points. In this schematic example there are 3 initial conditions and 3 randomly seeded AIMD simulations for each set of initial conditions. Fingerprinting: The results of simulations for each set of initial conditions are averaged across the final equilibrated 1000 AIMD steps of the simulation, and the ratios of coordination environments are compared between the simulation and experiment. Average over configurations which match experimental data are then used for Building the Model. The model consists of a total of 45 randomly selected configurations ( 15 from each individual AIMD simulation out of the 3 randomly seeded AIMD simulations). This model is collated and shown as the purple squares labeled 'amorphous model'. These 45 configurations are then used in the Experimental Validation in which the total observable is calculated as an average over static configurations in the amorphous model. 

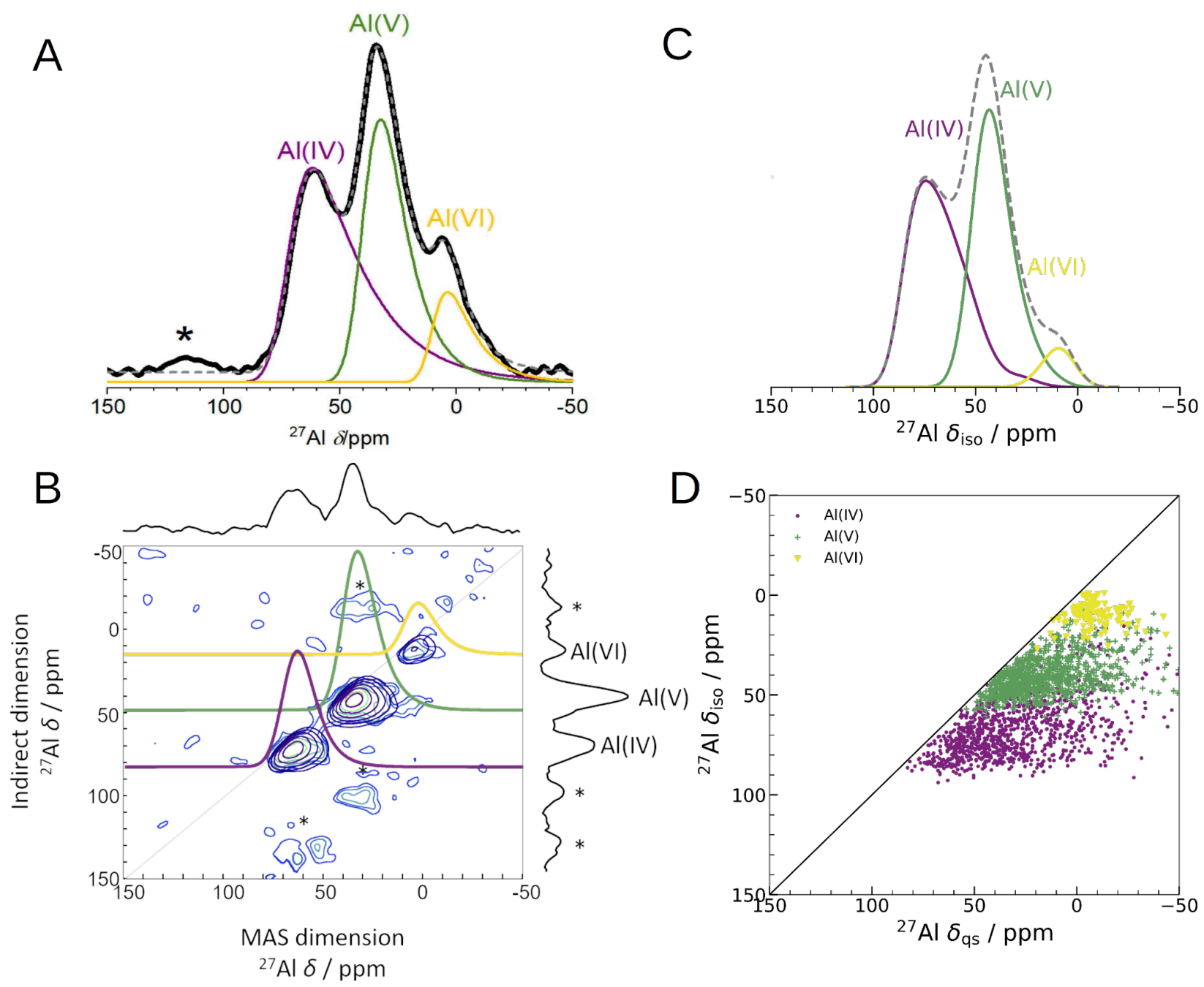

Fig. 2: Experimental 1D NMR and MQMAS compared to isotropic shieldings from computed NMR from DFT. (A) 1D ${ }^{27} \mathrm{Al}$ experimental NMR (black) with three fits using the Czjzek model (solid lines; GIM case (see Experimental Methods)) a model commonly applied to spectra of quadrupolar nuclei such as ${ }^{27} \mathrm{Al}$ when the materials are disordered (23). The fits are summed to produce overall 1D spectra (dashed grey) of ALD deposited $\mathrm{Al}_{2} \mathrm{O}_{3}$. Spinning sidebands are indicated with an asterisk. Each signal is colored according to the closest geometric environment, based on experimental shift values for Al(IV,V,VI). (B) MQMAS of ALD deposited $\mathrm{Al}_{2} \mathrm{O}_{3}$ shows that $\mathrm{Al}(\mathrm{IV})$ and $\mathrm{Al}(\mathrm{V})$ both have large quadrupolar shifts in the MAS dimension. (C) GIPAW NMR calculated spectra of the amorphous model. The distributions of $\mathrm{Al}$ environments are shown and broadening of $8 \mathrm{ppm}$ is applied. Note that this $\mathrm{X}-$ axis is $\delta_{\text {iso }}$ indicating that this is a plot of the isotropic shifts from each individual Al atom, rather than the quadrupolar lineshapes (shown in Fig. 3). This corresponds to the spectrum at an infinitely high magnetic field. (D) Comparison of $\delta_{i s o}$ and $\delta_{q s}$ from GIPAW NMR where the second-order quadrupolar shift $\left(\delta_{q s}\right)$ is calculated using the method from (24). This method again shows distortions in the quadrupolar dimension for both $\mathrm{Al}(\mathrm{IV})$ and $\mathrm{Al}(\mathrm{V})$, which is consistent with the MQMAS in $\mathbf{B}$. The distribution of $\delta_{q s}$ indicates that quadrupolar effects play a large role in the experimental 1D lineshape. 
A

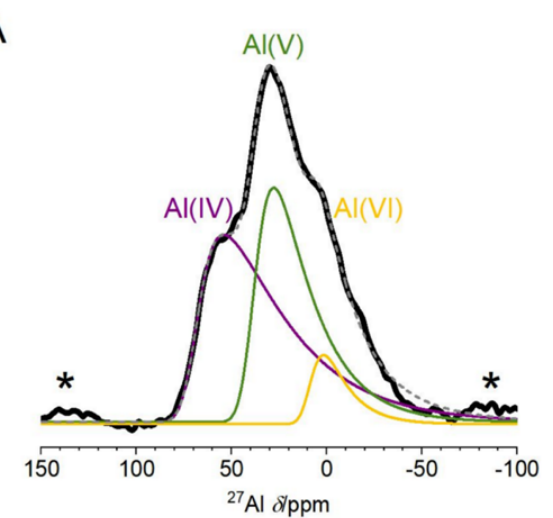

B

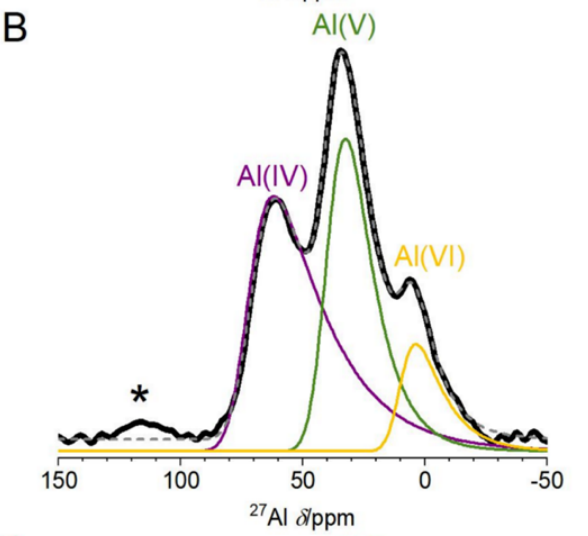

C

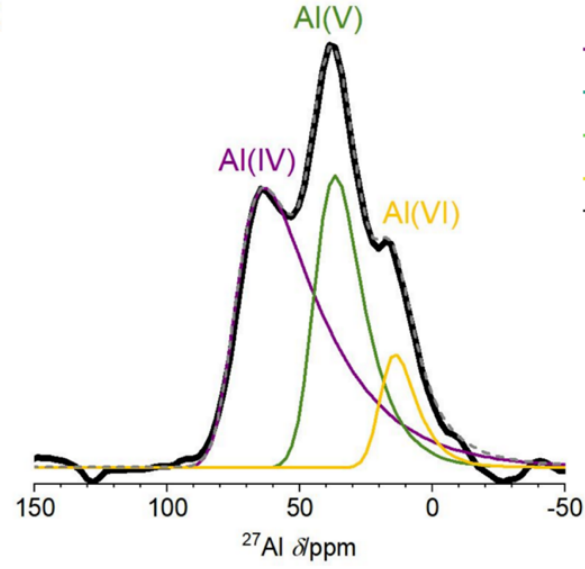

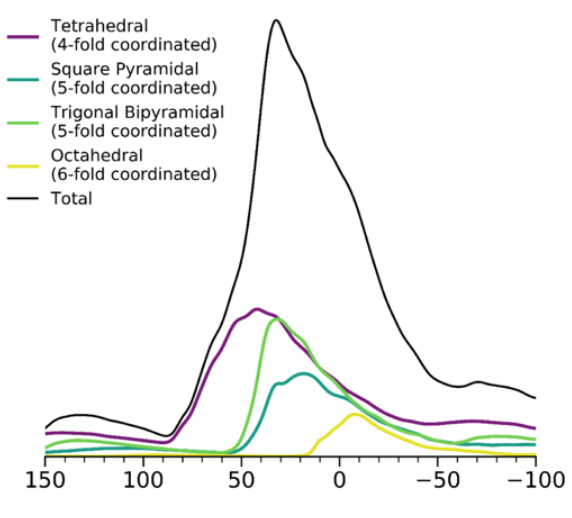
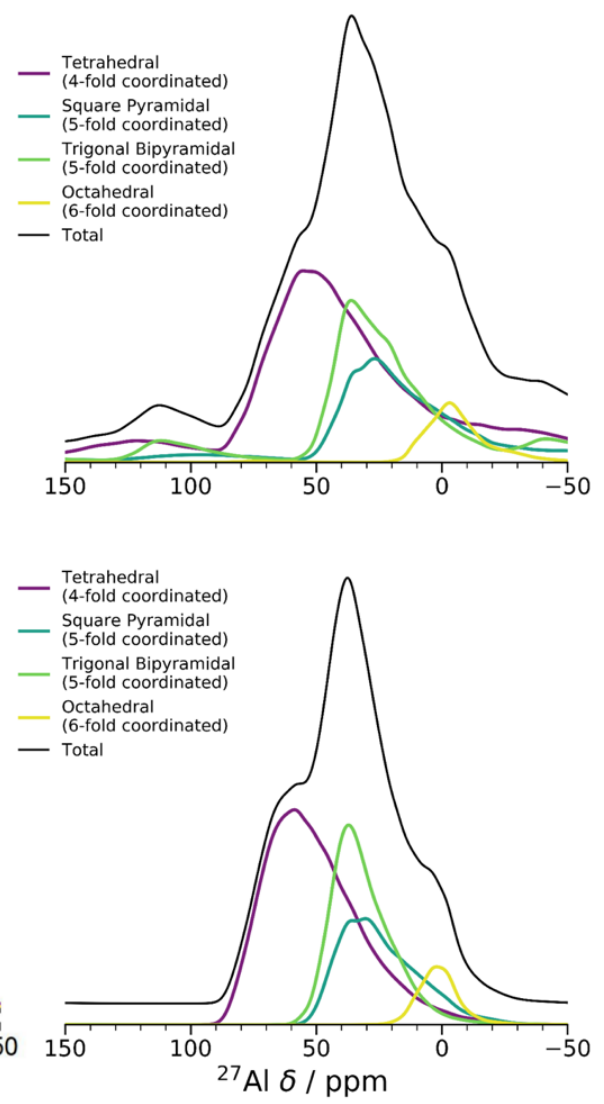

Fig. 3: 1D ${ }^{27}$ Al NMR at 3 fields (A 11.75 T, B 16.44 T, C 23.49 T) experimental and computed spectra. Left panel is experimental spectra, the right panel is calculated spectra with quadrupolar effects calculated using SIMPSON (17). Two lower field spectra were measured using a one pulse sequence, and the high field spectrum was measured using a Hahn-echo pulse sequence. Spinning sidebands are marked with asterisks in the experimental spectra. Experimental fitting was obtained using a Czejek model. DFT-calculated spectra show Al(V) lineshapes separated into square pyramidal and trigonal bipyramidal environments. These calculated spectra represent the first known DFT-calculated NMR lineshapes of amorphous materials and show excellent comparison to the experimental spectra. At higher fields in both cases the three peaks (Al(IV,V,VI)) become more pronounced, and are easily identifiable as separate environments. 


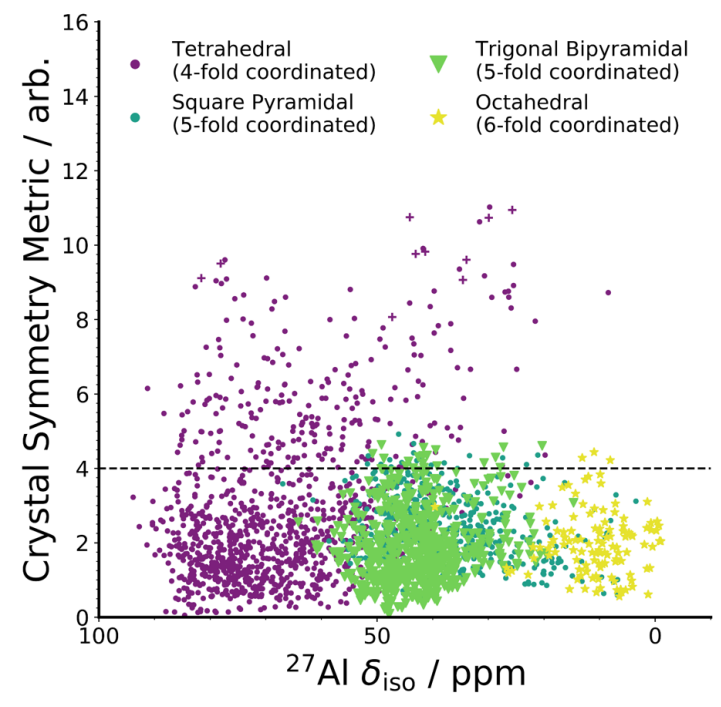

Fig. 4: DFT calculated isotropic shielding compared to the distortion of its geometric environment. GIPAW NMR calculated $\delta_{i s o}$ versus CSM as defined by ChemEnv (18). Each coordination environment is broken down into its closest geometric environment and coordination number: Al(IV) sites were classified as tetrahedral, Al(V) sites were subdivided into trigonal bipyramidal and square pyramidal and $\mathrm{Al}(\mathrm{VI})$ as octahedral. There are a large number of distorted $(\mathrm{CSM}>4)$ tetrahedral environments, which have a range of $\delta_{i s o}$ shifts. 


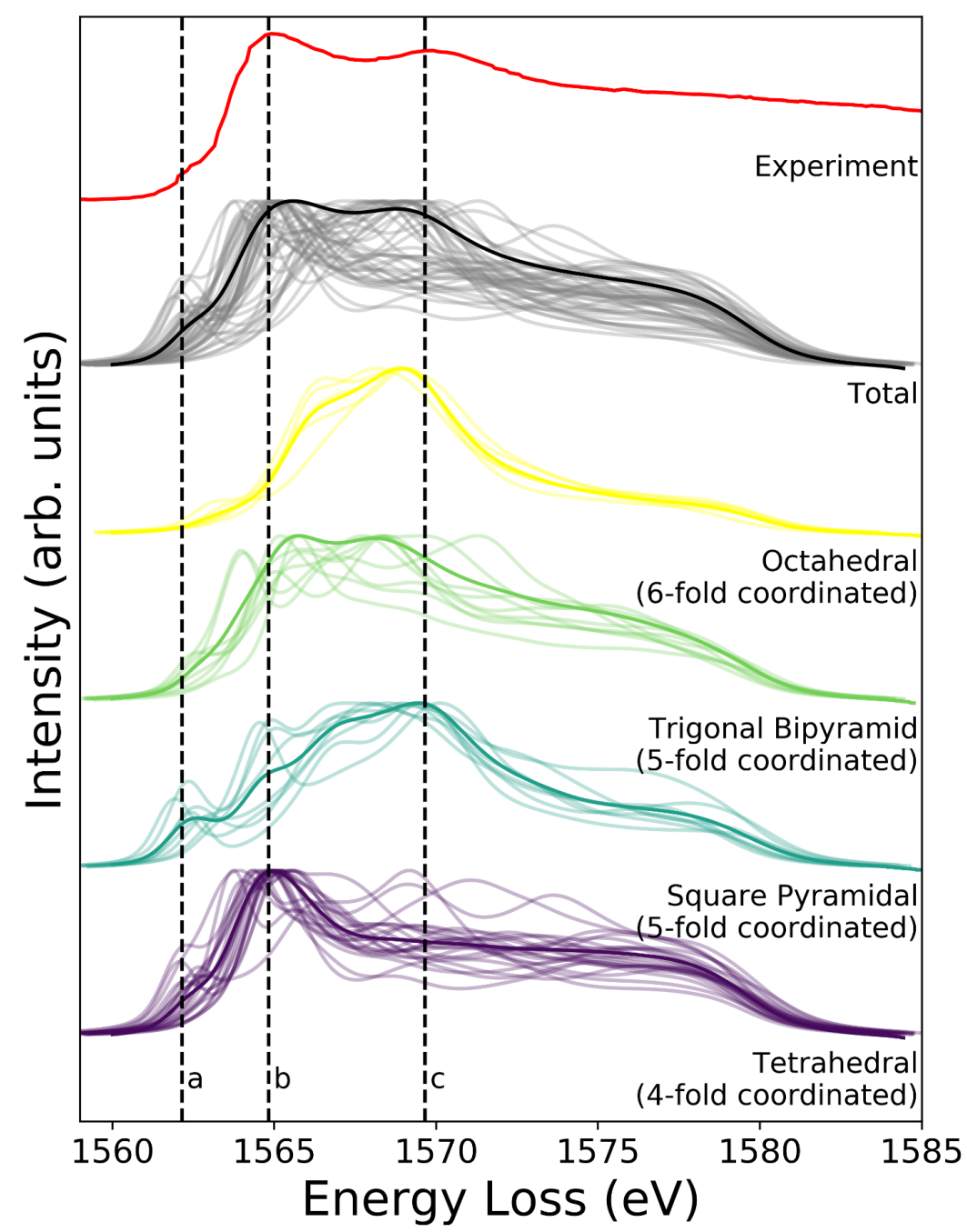

Fig. 5: Experimental XAS spectra obtained from the ALD deposited sample compared to the calculated core-hole XAS. The experimental spectra is identified by the red line labeled Experiment, and is obtained from the 1000 layer ALD deposited sample. Three distinct features at 1562,1565 , and $1570 \mathrm{eV}$ are denoted by dashed lines a, b, and c. The grey lines in the Total spectra show each individual spectra calculated at a single Al site in each configuration from the model, and the solid black line is the sum of those spectra. DFT calculated spectra separated by coordination environment are shown in the bottom four spectra; thin lines are individual spectrum, thick lines are the sum of each geometric site's spectra. All Al(IV) sites were classified as tetrahedral, $\mathrm{Al}(\mathrm{V})$ sites were subdivided into trigonal bipyramidal and square pyramidal, and $\mathrm{Al}(\mathrm{VI})$ as octahedral. Dashed vertical lines indicate relevant peaks in the experimental spectra. 


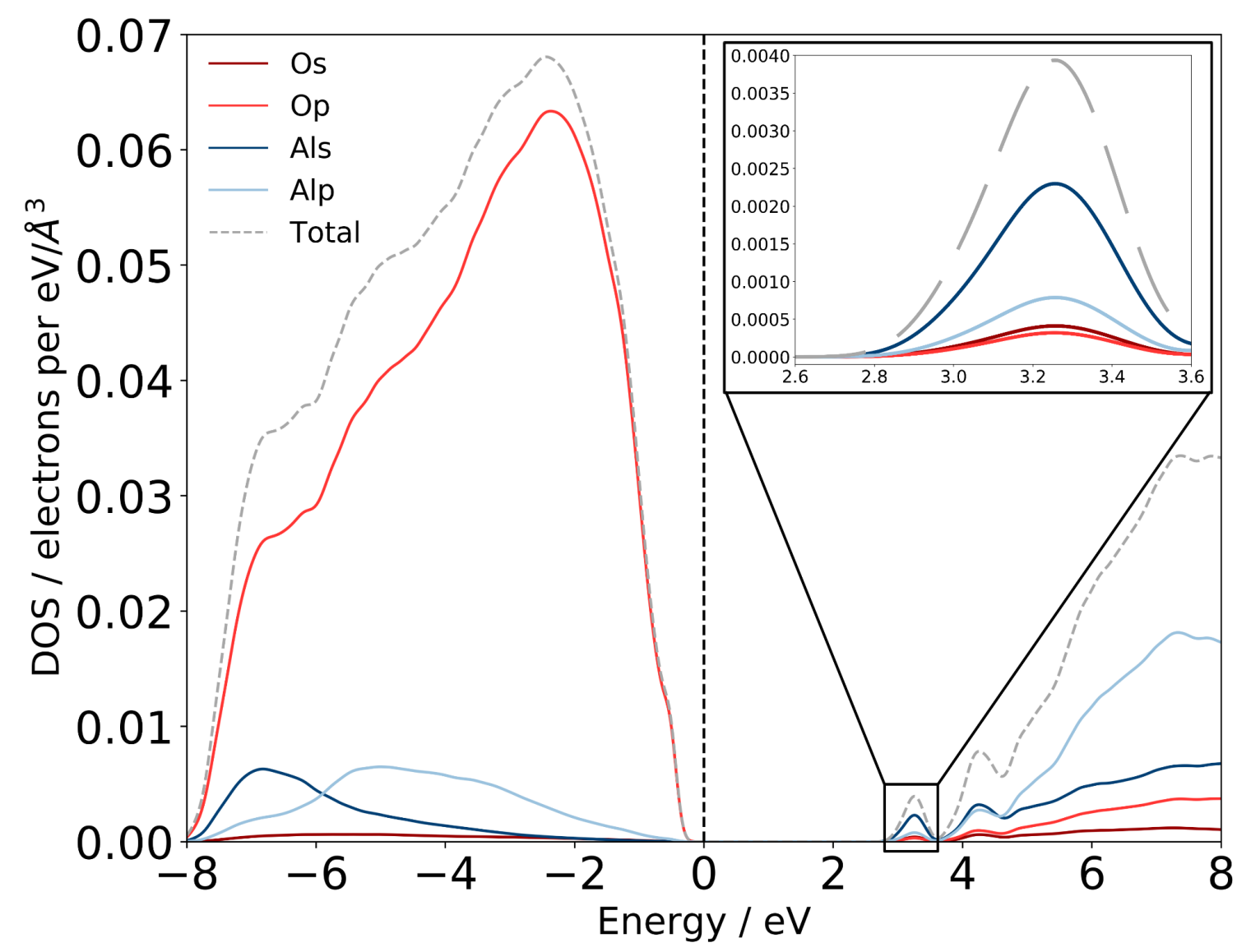

Fig. 6: Computed electronic density of states for amorphous alumina. Total electronic density of states separated by atom and orbital contribution shows that $\mathrm{a}-\mathrm{Al}_{2} \mathrm{O}_{3}$ is a wide bandgap insulator, with a gap of $2.6 \mathrm{eV}$. States at the top of the valence band, near the fermi level are primarily $\mathrm{O} p$ states, with states in the conduction band minimum being primarily Al $s$ (detail shown inset top right). The dashed grey line is the sum of these states, and the total density of states was broadened using a Gaussian broadening scheme of width $0.1 \mathrm{eV}$, as implemented in OptaDOS $(25,26)$. The Fermi level is set to $0 \mathrm{eV}$ for all configurations. Two localised states at 3.2 and $4.2 \mathrm{eV}$ above the Fermi level have $\mathrm{Al} s$ character and mixed Al $s, p$ character, respectively. 